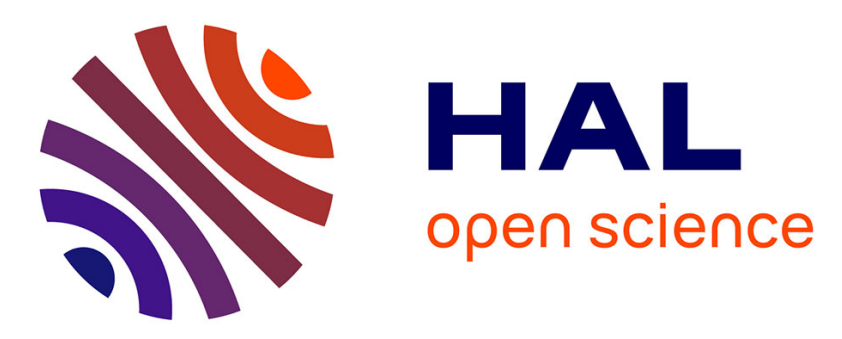

\title{
A Co-Evolutionary Approach to Analyzing the Impact of Rationality on the Italian Electricity Market
}

Célia da Costa Pereira, Sara Bevilacqua, Eric Guerci, Frédéric Precioso, Claudio Sartori

\section{- To cite this version:}

Célia da Costa Pereira, Sara Bevilacqua, Eric Guerci, Frédéric Precioso, Claudio Sartori. A CoEvolutionary Approach to Analyzing the Impact of Rationality on the Italian Electricity Market. PRIMA 2019: Principles and Practice of Multi-Agent Systems - 22nd International Conference, Turin, Italy, October 28-31, 2019, Proceedings, Oct 2019, Turin, Italy. pp.235-250. hal-03199500

\author{
HAL Id: hal-03199500 \\ https://hal.science/hal-03199500
}

Submitted on 15 Apr 2021

HAL is a multi-disciplinary open access archive for the deposit and dissemination of scientific research documents, whether they are published or not. The documents may come from teaching and research institutions in France or abroad, or from public or private research centers.
L'archive ouverte pluridisciplinaire $\mathbf{H A L}$, est destinée au dépôt et à la diffusion de documents scientifiques de niveau recherche, publiés ou non, émanant des établissements d'enseignement et de recherche français ou étrangers, des laboratoires publics ou privés. 


\title{
A Co-Evolutionary Approach to Analyzing the Impact of Rationality on the Italian Electricity Market
}

\author{
Célia da Costa Pereira ${ }^{\star}$, Sara Bevilacqua ${ }^{2}$, Eric Guerci ${ }^{3}$, Frédéric Precioso ${ }^{1}$, \\ and Claudio Sartori ${ }^{2}$ \\ 1 Université Côte d'Azur, CNRS, I3S Lab. \\ celia.pereira,frederic.precioso@unice.fr \\ 2 Università di Bologna \\ sara.bevilacqua, claudio.sartori@unibo.it \\ ${ }^{3}$ Université Côte d'Azur, CNRS - GREDEG \\ eric.guerci@unice.fr
}

\begin{abstract}
We analyze the behavior of the Italian electricity market with an agent-based model. In particular, we are interested in testing the assumption that the market participants are fully rational in the economical sense. To this end, we suppose that while constructing its strategy the agent takes into account all the possible strategies the other (competitors) agents might adopt in the future, not only their last strategies, as it is done in the literature. This motivates us to propose a coevolutionary approach to strategy optimization, which better reflects the way actual decision makers behave in reality. The experiments carried out corroborate our hypothesis and show an improvement in the results compared to the literature.
\end{abstract}

\section{Introduction}

The need for understanding the evolution of the prices in the electrical power markets has increased with the new trends of the electrical market in many countries $[15,14]$. Artificial Intelligence techniques have already proven to be effective in modeling the electricity market. Faia and colleagues proposed in [4] a Genetic Algorithm (GA) based approach to solve the portfolio optimization problem for simulating the Iberian electricity market. The results show that their GA based method is able to reach better results than previous implementations of Particle Swarm Optimization (PS) and Simulated Annealing (SA) methods. They also compared their results with the ones obtained with a deterministic approach. Santos and colleagues proposed in [13] a new version of the MultiAgent System for Competitive Electricity Markets (MASCEM, [12]) with the aim of optimizing it with repect to the results as well as to the execution time, in order to face the highly demanding requirements from the decision support.

* Célia da Costa Pereira acknowledges support of the PEPS AIRINFO project funded by the CNRS. 
Other models have been proposed, like the one presented by Urielli and colleagues [18], in which the authors study the impact of the Time-Of-Use (TOU) tariffs in a competitive electricity marketplace. A very interesting and recent survey of potential design changes in the electricity market and their consequences, has been proposed by Ela and colleagues in [3].

In this paper, we propose a framework which helps analysing the behavior of the participants in the Italian electrical power market [16]. We would like to stress that our interest is in understanding how the market behaves as a consequence of the actions of its participants to make profit, and also in analysing the behavior of the market in order to maximize the social welfare from an economical rational point of view $[19,17]$, i.e., with respect to the electricity producers as well as with respect to the electricity consumers. To this aim, we build upon our previous contribution [2], which reproduced and then extended an existing economical-based model of the Italian electricity market [8]. Instead of treating the strategies of the market competitors as already known (from the previous auctions) and fixed, here we adopt a more sophisticated setting, more in line with reality, whereby each market participant optimizes its strategy while trying to anticipate the strategies its competitors could adopt. This led us to devise a co-evolutionary approach to strategy optimization.

The paper is organized as follows. Section 2 briefly presents the three optimization methods used in the paper. Section 3 presents the mechanism of exchanges in the Italian market proposed in the literature. Section 4 presents the problem statement and Section 5 discribes the co-evolutionary approach as well as the obtained results. Section 6 concludes the paper.

\section{Some Background: A Brief Description of the Used Methods}

In this section, we will briefly present the three methods used in our work to model the rationality of the market participants.

A Genetic Algorithm (GA) [9,7] is a computational technique inspired by biology. The basic idea of a GA is to mimic the Darwinian principle of survival of the fittest, according to which species with a high capacity of adaptation have a higher probability to survive and then to reproduce. The algorithm considers a population of individuals represented by their genes. Three operators can be used to mimic the evolution of these individuals: mutation, which randomly changes some bits of a gene, crossover, which mimics the sexual reproduction of the living beings, and selection, which consists of deciding which among the individuals in the population will survive in the next generation. This choice is made thanks to a fitness function, which is an objective function allowing to compute the extent to which an individual of the population is adapted to solve the considered problem.

In Monte Carlo Optimization [1], an approximation to the optimum of an objective function is obtained by drawing random points from a probability distribution, evaluating them, and keeping the one for which the value of the 
objective function is the greatest (if a maximum is sought for) or the least (if a minimum is sought for). As the number of points increases, the approximation converges to the global optimum.

Particle Swarm Optimization (PS) $[11,10]$ is a meta-heuristic method inspired by the behavior or rules that guide groups of animals, for example bird flocks. According to these rules, the members of the swarm need to balance two opposite behaviors in order to reach the goal: individualistic behavior, in which each element searches for an optimal solution, and social behavior, which allows the swarm to be compact. Therefore, individuals take advantage from other searches moving toward a promising region. In this algorithm, the evolution of the population is re-created by the changing of the velocity of the particles. The idea is to tweak the values of a group of variables in order to make them become closer to the member of the group whose value is closest to the considered target. PS is similar to genetic algorithms (GAs). It is also a population-based method with the particularity that the elements of the population are iteratively modified until a termination criterion is satisfied.

\section{The Italian Electricity Market}

\subsection{The Market Configuration}

The reality of the Italian Electricity Market which takes place in the Italian Power Exchange (IPEX), considers a two-settlement market configuration with a generic forward market and the Day-Ahead Market (DAM). The DAM price value is commonly adopted as underlying for forward contracts; therefore, as in Guerci et al. [8], we will refer to DAM as the spot (i.e., immediate, instantaneous) market session for simplicity. The forward market session is modeled by assuming a common, zone-independent, and unique forward market price $P^{f}$ for all market participants and by determining the exact historical quantity commitments for each generating unit.

\section{Definition 1 (Generating Company).}

A generating company (GenCo) is an agent $g$, (with $g=1,2, \ldots, G$, and $G$ is the number of GenCos) which owns $N_{g}$ generators $^{4}$. The ith generator (where $i=1,2, \ldots, N_{g}$ ) has lower $\underline{Q}_{i, g}$ and upper $\bar{Q}_{i, g}$ production limits, which define the feasible production interval for its hourly real-power production level in $M W$ (Mega Watt) $\hat{Q}_{i, g, h}=\hat{Q}_{i, g, h}^{f}+\hat{Q}_{i, g, h}^{s}$, with $\underline{Q}_{i, g} \leq \hat{Q}_{i, g, h} \leq \bar{Q}_{i, g}$ where $\hat{Q}_{i, g, h}^{f}$ and $\hat{Q}_{i, g, h}^{s}$ are respectively the quantity sold in the forward market and the quantity accepted in the DAM in each hour $h$.

It is assumed that the company $g$ takes a long position in the forward market (it means that the company makes agreement with the market operator with large advance) for each owned generator $i$, corresponding to a fraction $f_{i, g, h}$ (where $h$ indicates the hour of the day) of its hourly production capacity, that

\footnotetext{
${ }^{4}$ In the following we will use the terms generator and power plant interchangeably.
} 
is $\hat{Q}_{i, g, h}^{f}=f_{i, g, h} \cdot \bar{Q}_{i, g}$. The value of such fraction varies throughout the day, indeed forward contracts are commonly sold according to standard daily profiles. The value of $f_{i, g, h}$ has been estimated by looking at historical data and thus corresponds to a realistic daily profile for each generator.

Definition 2 (Revenues for the forward and spot markets). The revenue in Euro per hour ([€h]), $R_{g, h}^{f}$, from forward contracts for company $g$ and given the unique forward market price $P^{f}$ is:

$$
R_{g, h}^{f}=\sum_{i=1}^{N_{g}} \hat{Q}_{i, g, h}^{f} \cdot P^{f}
$$

The spot revenue, $R_{g, h}^{s}$, per hour for GenCo $g$ is obtained as follows:

$$
R_{g, h}^{s}=\sum_{z=1}^{Z} \hat{Q}_{z, g, h}^{s} \cdot P_{z, h}^{s}
$$

where $P_{z, h}^{s}$ is the price in the spot market in zone $z$ at hour $h$, and $Z$ is the total number of zones.

Let $C_{i, g, h}([€ / \mathrm{h}])^{5}$ be the total cost (of production) function of the $i^{\text {th }}$ generator of GenCo $g$. The total profit per hour, $\pi_{g, h}$, [€/h] for GenCo $g$ is computed as follows:

$$
\pi_{g, h}=R_{g, h}^{s}+R_{g, h}^{f}-\sum_{i=1}^{N_{g}} C_{i, g, h}\left(\hat{Q}_{i, g, h}\right)
$$

The considered set of thermal power plants, independently owned by GenCos, consists of up to 224 generating units, using 5 different technologies. The number of generation companies and generating units offering in the DAM varies throughout the day. Based on historical data, it has been determined for each period (day and hour) the thermal power plants that offered in DAM. ${ }^{6}$ For each power plant in the dataset, information on the maximum and minimum capacity limits is available, as well as on the parameters needed to compute the cost.

\subsection{Market Exchanges}

A GenCo $g$ submits to the DAM a bid consisting of a pair of values corresponding to the limit price $P_{i}^{s}([€ / \mathrm{MW}])$ and the maximum quantity of power $Q_{i}^{s} \leq$ $\bar{Q}_{i, g}-\hat{Q}_{i, g}^{f}([\mathrm{MW}])$ that it is willing to be paid and to produce, respectively. After receiving all generators bids, the market operator clears the DAM by performing a social welfare maximization, subject to the following constraints:

\footnotetext{
5 The details about the function can be found in [8].

${ }^{6}$ Notice that bid data are publicly available on the power exchange website with a one-week delay, therefore, information about what plants were actually present and the like is supposed to be common knowledge.
} 
- the zonal energy balance (Kirchhoff's laws),

- the maximum and minimum capacity of each power plant,

- the inter-zonal transmission limits.

It is worth noting that the Italian demand curve in the DAM is price-inelastic, i.e., it is unaffected when the price changes. Therefore, the social welfare maximization can be transformed into a minimization of the total reported production costs, i.e., of the bid prices (see Equation 4). This mechanism determines both the unit commitments for each generator and the Locational Marginal Price (LMP) for each connection bus. However, the Italian market introduces two slight modifications. Firstly, sellers are paid the zonal prices (LMP), therefore, this fact has to be explicitly considered in the model, whereas buyers pay a unique national price (PUN, for Prezzo Unico Nazionale) common for the whole market and computed as a weighted average of the zonal prices with respect to the zonal loads. Secondly, transmission power-flow constraints differ according to the flow direction.

The factor to minimize by solving the linear program is the following:

$$
\min \sum_{g=1}^{G} \sum_{i=1}^{N_{g}} P_{i, g, h}^{s} \hat{Q}_{i, g, h}^{s},
$$

which is subject to the following constraints:

- Active power generation limits: $\underline{Q}_{i, g} \leq \hat{Q}_{i, g, h}=\hat{Q}_{i, g, h}^{s}+\hat{Q}_{i, g, h}^{f} \leq \bar{Q}_{i, g}[\mathrm{MW}]$

- Active power balance equations for each zone $z$ :

$\sum_{g=1}^{G} \sum_{j \in z} \hat{Q}_{j, g, h}^{s}-Q_{z, l o a d, h}=Q_{z, \text { inject }, h}[\mathrm{MW}]$

being $\sum_{g=1}^{G} \sum_{j \in z} \hat{Q}_{j, g, h}^{s}$ the sum of all the productions over all generators located in zone $z, Q_{z, l o a d, h}$, the load demand at zone $z$ in hour $h$ and $Q_{z, \text { inject }, h}$, the net oriented power injection in the network at zone $z$ in hour $h$.

- Real power flow limits of line, $l: Q_{l, s t} \leq \bar{Q}_{l, s t}[\mathrm{MW}]$ and $Q_{l, t s} \leq \bar{Q}_{l, t s}[\mathrm{MW}]$ being $Q_{l, s t}$ the power flowing from zone $s$ to zone $t$ of line $l$ and $\bar{Q}_{l, s t}$ the maximum transmission capacity of line $l$ in the same direction. $Q_{l, s t}$ are calculated with the standard DC power flow model [5].

The solution consists of the set of the active powers $\hat{Q}_{i, g, h}^{s}$ generated by each plant $i$ and the set of zonal prices $P_{z}^{s}$ (LMPs) for each zone $z \in[1,2, \ldots, Z]$, where $Z$ is the number of zones.

\section{Problem Statement}

In this section we will present a general statement of the problem of choosing the most competitive strategy for a GenCo. 


\subsection{Model description}

Each GenCo $g$ must submit to the DAM a bid, i.e., a set of prices for each of its own power plants. Therefore, each GenCo has an action space for each power plant, which is a set of possible prices that the GenCo can choose. This set is represented by vector $A S_{i, g}$, which is obtained with the following product:

$$
A S_{i, g}=M C_{i, g} \cdot M K \text { set },
$$

where $A S_{i, g}$ represents the action space of power plant $i$ of GenCo $g, M C_{i, g}$ is the marginal cost of the same power plant, and $M K$ set $=[1.00,1.04, \ldots, 5.00]$ is the vector with the mark-up levels. In this way, GenCos are sure not to propose a price lower than the costs.

The Multi-Agent System The multi-agent system is depicted in Figure 1. The $G$ GenCos are reported on the top of the figure. These GenCos repeatedly interact with each other at the end of each period $r \in\{1, \ldots, R\}$, that is they all submit bids to the DAM according to their current beliefs on opponents strategies. At the beginning of each period $r$, GenCos need to study the current

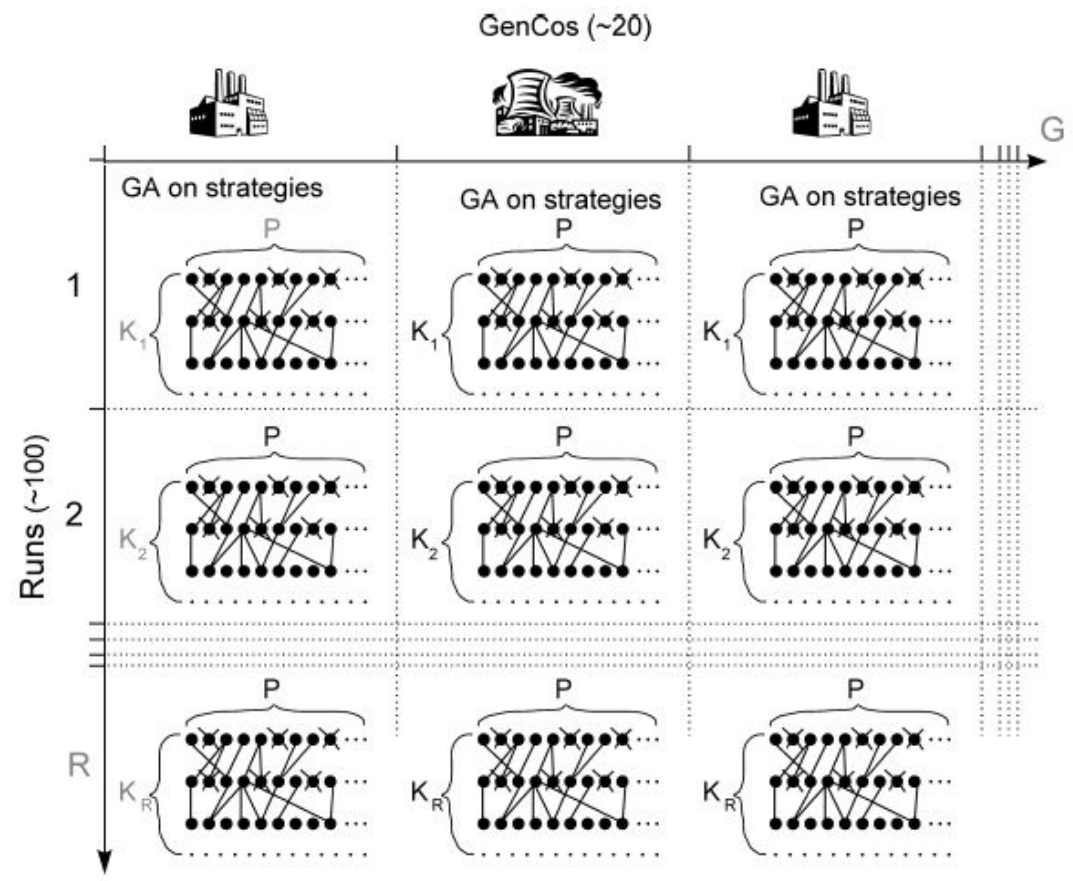

Fig. 1. A schematic representation of a simulation. 
market situation in order to predict which strategies their competitors will adopt and identify the best reply to their opponents, to be played at period $r+1$.

In order to choose the most competitive strategy, GenCos need to repeatedly solve the market for different private strategies and predicted strategies of their opponents, much in the same way as a chess player has to anticipate all possible moves of her/his opponent before deciding which move to play. This correponds to an optimization problem.

\subsection{The Optimization Process}

The general purpose of the optimisation algorithm is to keep a large population of candidate strategies and to improve at the same time their fitness/performance in the market. Thus, a population of size $P$, (see Figure 1), of strategies is defined, which evolves throughout the $K_{r}$ generations.

Strategy A strategy is a vector of prices in the action space, one for each of the $N_{g}$ power plants of GenCo $g$ (depicted as a black dot in Figure 1).

Profit The profit generated by GenCo $g$ using strategy $x$, while its competitors are using the strategies collectively represented by $y$ (a vector whose length is $\sum_{g^{\prime} \neq g} N_{g^{\prime}}$ ) can be computed based on Equation 3. In the following, it will be convenient to denote such profit as profit $(x, y)$.

Selecting a Strategy At the end of each period $r$, each GenCo bids to the market by selecting one strategy among its current population of candidates.

In [8] the selection is done according to a probabilistic choice model in order to favor the most represented strategy in the population (i.e., based on the frequency probability). In addition to the frequency based strategy of selection, in [2] a second strategy is used, based on the value of the fitness of the individuals, named fitness-based strategy. Here, like in [2], we also consider these two strategies.

\section{Intelligent GenCos: A Co-Evolutionary Approach}

In this section, we will present an extension of the framework proposed in [2]. We will start with a brief explanation of our motivations and then we will present the methodology we have adopted.

\subsection{Motivations}

In previous work, like [8] and [2], it is supposed that the GenCos share their strategies: a GenCo constructed its strategy while making the hypothesis that the other GenCos maintain their last strategies. More precisely, the populations of strategies evolve separately and the profit of a GenCo depends on the strategies of the other GenCos. In particular, each GenCo considers, at time $t$, the 
strategies adopted by its competitors at time $t-1$, not the ones it would expect its competitors to adopt at time $t$. In other words, the expectation of a GenCo is that all the competitors will repeat the strategy they used at the previous auction.

The framework we are proposing here allows instead to consider more realistic situations. The hypothesis we make is that each GenCo should consider, during the period in which it contructs its strategy, all the possible strategies the other GenCos might adopt. This way, the strategy under construction can be optimized against the most unfavorable competitors' current strategies.

This approach may be seen as a kind of adversarial reasoning. The difference is that in the game reasoning framework, the goal is to win against the opponent, but here there are no winners and losers: the goal for a GenCo is to obtain high profits as much as possible for its own characteristics and possibilities, while respecting all the constraints imposed by the market. Therefore, under this assumption, it is possible that the best strategy for a GenCo allows a competitor to obtain a higher profit. In what follows, we will notice that the results of the experiments made using this new framework for one particular configuration outperform the previous results, but we will first explain the fundamental ideas underlying our proposals.

\subsection{Methodology}

Here, instead of having $G$ (where $G$ is the number of GenCos) updating algorithms evolving with one different population for each GenCo, our proposal is to evolve $G$ updating algorithms with two populations for each GenCo: the first population concerns the GenCo's own strategy, while the second population concerns the possible strategies of all the other GenCos. Therefore, the individuals of the second population represent the strategies of the remaining $G-1$ GenCos.

Benefits Two benefits emerge from considering two populations. The first one is due to the introduction of the competitive aspect in the process. The second one is due to the fact that we can now account for the independence between GenCos unlike in [2], where GenCos were supposed to share their strategy with their competitors in order to allow for the evolution of the population. Our proposal is more in line with the reality of the Italian market, in which the companies do not share their strategies with competitors. Therefore, by adding a second population, GenCos can avoid sharing these precious information and they can reason by themselves.

Figure 2 illustrates the two populations: the GenCo's own population on the left-hand side and the population of its competitors on the right-hand side. Let $x_{i}$ be one individual of Population 1 (red rectangle) and let $y_{j}$ be one individual of Population 2 (blue rectangle). The fitness of an individual $x_{i}$ of Population 1, $f^{+}\left(x_{i}\right)$, is to be maximized while the fitness of an individual of Population 2, $f^{-}\left(y_{j}\right)$, is to be minimized. $f^{+}\left(x_{i}\right)$ may correspond to the average of the profit that the GenCo would obtain if it adopts this strategy $\left(x_{i}\right)$ by considering all the possible strategies $y_{j}$ of its competitors: 


\section{Genco $g$}
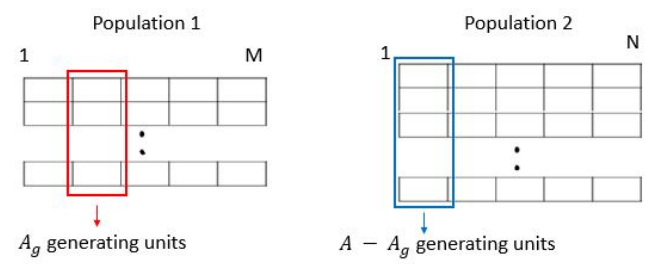

where $A$ is the total number of generating units

Fig. 2. Schematic representation of the two populations of a GenCo

$$
f^{+}\left(x_{i}\right)=\frac{1}{N} \cdot \sum_{j=1}^{N} \operatorname{profit}\left(x_{i}, y_{j}\right),
$$

where $N$ in the size of Population 2 and profit is the function that estimates the profit of GenCo $g$, given all generating units bids. The objective is to maximize the fitness $f^{+}\left(x_{i}\right)$ for each $x_{i}$ in Population 1. Many other approaches can be adopted, for example by considering the different possible variations when computing the fitness. One particular example is to use the minimum instead of the average as follows:

$$
f^{+}\left(x_{i}\right)=\min _{j=1 \ldots N}\left(\operatorname{profit}\left(x_{i}, y_{j}\right)\right)
$$

In this case, the best strategy in Population 1 corresponds to the most robust strategy, i.e., the one which guarantees the highest profit against the strongest competitor.

However, this new proposal increases considerably the execution time. Indeed, instead of solving a linear programming problem $M$ times, we have now $N M$ linear programming problems to solve.

Proposed solution In order to reduce the execution time, we have considered only the best strategy among the competitors' strategies. The best strategy for the agent will then be the one which allows the GenCo to achieve better results against the best of the competing strategies:

$$
f^{+}\left(x_{i}\right)=\operatorname{profit}\left(x_{i}, y_{\text {best }}\right)
$$

where $y_{\text {best }}$ is the element of Population 2 with the lowest fitness or the element with the highest frequency. In this case, the linear programming problem is solved $M$ times.

It should be recalled that our aim is to find the most robust strategy for the GenCo, i.e., the strategy which best replies to the competitors even if they all do their best to minimize the GenCo's profit. As for the fitness of individuals 
of Population 1, several possibilities can be considered to compute the fitness of individuals of Population 2:

- Compute the fitness as an average:

$$
f\left(y_{j}\right)=\frac{1}{M} \cdot \sum_{i=1}^{M} \operatorname{profit}\left(x_{i}, y_{j}\right)
$$

where $M$ is the size of Population 1.

- Choose the strategy with the maximum profit among all the possibilities:

$$
f\left(y_{j}\right)=\max _{i=1 \ldots M}\left(\operatorname{profit}\left(x_{i}, y_{j}\right)\right)
$$

- Estimate the fitness considering only the best strategy of Population 1, in order to reduce the computational effort of the two previous cases (from $N M$ to $N)$ :

$$
f\left(y_{j}\right)=\operatorname{profit}\left(x_{\text {best }}, y_{j}\right)
$$

where $x_{\text {best }}$ is the element of Population 1 with the highest fitness value or with the highest frequency.

Many scenarios can be explored by mixing different approaches of computing the fitness from the two populations. Accordingly, the computational effort varies: in the best case (using equations 8 and 11) the LP problem is solved $N M$ times and in the worst case it is solved $(N M)^{2}$ times per iteration. Taking into account the generations of the genetic algorithm $n_{\text {gen }}$, the computational efforts become, respectively, $n_{\text {gen }} \cdot N M$ and $\left(n_{\text {gen }} \cdot N M\right)^{2}$ per iteration.

\subsection{Evaluation of the Proposed Approach}

To validate the proposed approach, we tested several combinations of fitness functions $\left(f^{+}\right.$and $\left.f^{-}\right)$, criteria for selecting one strategy from a population, and optimisation algorithms. The Hist line (red line) reports real values of the PUN. Two versions have been considered:

1. with $f^{+}$defined as in Equation 8, $f^{-}$as in Equation 11:

(a) GAfreq1 : frequency-based strategy and genetic algorithm;

(b) GAfitness $1: f^{+}$fitness-based strategy and genetic algorithm;

(c) PSfreq1: $f^{+}$frequency-based strategy and swarm optimization;

(d) PSfirness $1: f^{+}$fitness-based strategy and swarm optimization;

(e) Monte Carlo.

2. with $f^{+}$defined as in Equation 7, $f^{-}$as in Equation 9:

(a) GAfreq2 : frequency-based strategy and genetic algorithm;

(b) GAfitness 2 : fitness-based strategy and genetic algorithm;

(c) PSfreq $2: f^{+}$frequency-based strategy and swarm optimization;

(d) PSfirness $2: f^{+}$fitness-based strategy and swarm optimization;

(e) Monte Carlo.

The remaining configurations have been left for future work. 
Data The demand of energy for each zone is provided in a load matrix with the following information: a first column which contains the zones, the second which contains the maximum limit prices and the third column which contains the demand quantities of electricity.

All the characteristics of the power plants are collected in a structure with the following features:

- the names of the GenCos (for example ATEL, EDISON, ...),

- the names of the used technologies (coal, combined cycle gas turbine, ...),

- the prices of the fuels,

- information related to the Italian power plants: the columns indicates respectively the zone, maximum production quantity, minimum production quantity, coefficient $a$, coefficient $b$, coefficient $c$ (see 3), GenCo's id, technology index, and fuel index and power plant's id.

- the production quantity data from other power plants (i.e., not produced by the $\mathrm{GenCo}$ ).

The PUN historical values used in the experiments are public data which can be found in $[6]$.

Implementation and Results The implementions have been done in MATLAB R2017a with Optimization and Global Optimization toolboxes. Experiments were performed on a computer running Windows 7 and based on an IntelCoreTMi7-3610QM @2.30GHz microprocessor with 8 GB main memory.

In all the simulations, the number of GenCos participating in the market varies between 15 and 19, while the number of power plants for each GenCo varies between 1 and 90. The three optimization methods use the Matlab default parameters and are allocated the same number of objective function evaluations.

The execution time varies a lot between different versions, since the execution effort varies. With two populations of 10 individuals, the combinations Version 1:(a)-(e) take about 16 seconds for the GA per iteration, 20 seconds for the PS and 6 seconds for Monte Carlo. The combinations Version 2: (a)-(e) require about 180 seconds per iteration for the GA, 240 second for the PS, and 55 seconds for Monte Carlo. In all the figures below, the real situation is plotted in red with the label Hist.

Figure 3 shows the results of genetic algorithm, particle swarm optimization and Monte Carlo optimization of IntelligentGenCo in Version 1. GAfitness1 (lilac line) is still low, GAfreq2 (dark-blue line) and GAfitness2 (dark-green line) still overestimate the PUN (historical red line). PSfreq1 (light-blue line), PSfreq2 (yellow line) and PSfitness2 (brown line) are quite good (see also Figures 4 and 5), except for the overestimation in the off-peak hours. GAfreq1 (light-green line) is equivalent to the previous ones, but it overestimates the PUN also in peak hours. PSfitness1 (orange line) is again the best algorithm, since it is able to reproduce the PUN in off-peak hours. The main difference is in Monte Carlo (dark dashed line with crosses), which has basically the same trend of GAfitness1 in this version. 


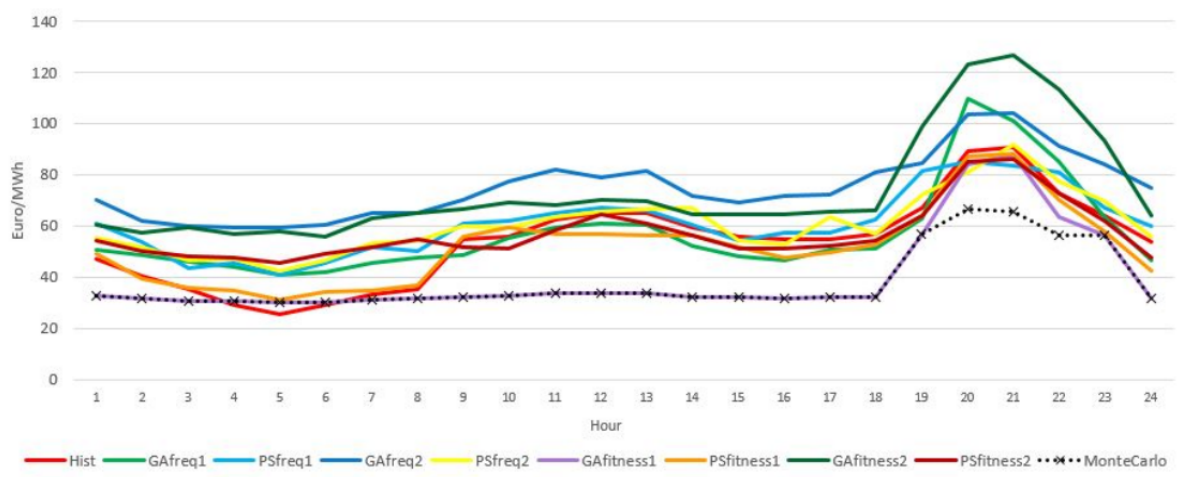

Fig. 3. Simulated PUNs for 2010-10-03, provided by IntelligentGenCo in Version 1

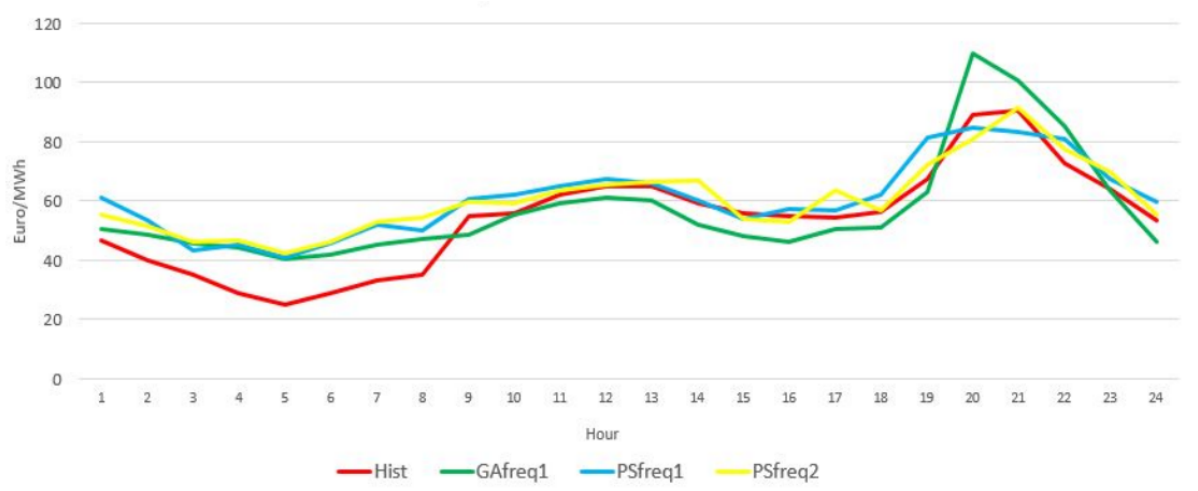

Fig. 4. Focus on the best frequency-based algorithms seen in Figure 3.

Figure 6 shows the results for the IntelligentGenCos Version 2. In this version results are very different with respect to the previous cases: now no line clearly overestimates the historical values (red line), except in off-peak hours. This result could be a consequence of the adversarial behavior of GenCos. In Figure 6, the lines relevant to the various algorithms lie very close to each other, especially in the central hours. Thus, the following detailed figures will focus on similar lines.

GAfitness1 (lilac line) is still low. Both PSfitness1 (orange line) and PSfitness2 (brown line) are low in off-peak hours, almost reaching the historical line (see Figure 7).

The remaining genetic algorithms GAfreq1 (light-green line), GAfreq2 (darkblue line) and GAfitness2 (dark-green line) are very close to each other, but only the last one is able to reach the peaks at $8 \mathrm{pm}$ and $9 \mathrm{pm}$ (as it could be seen in Figure 8 ). The last three algorithms are very close to the historical line in the central hours, but they overestimate the off-peak hours (see Figure 9). 


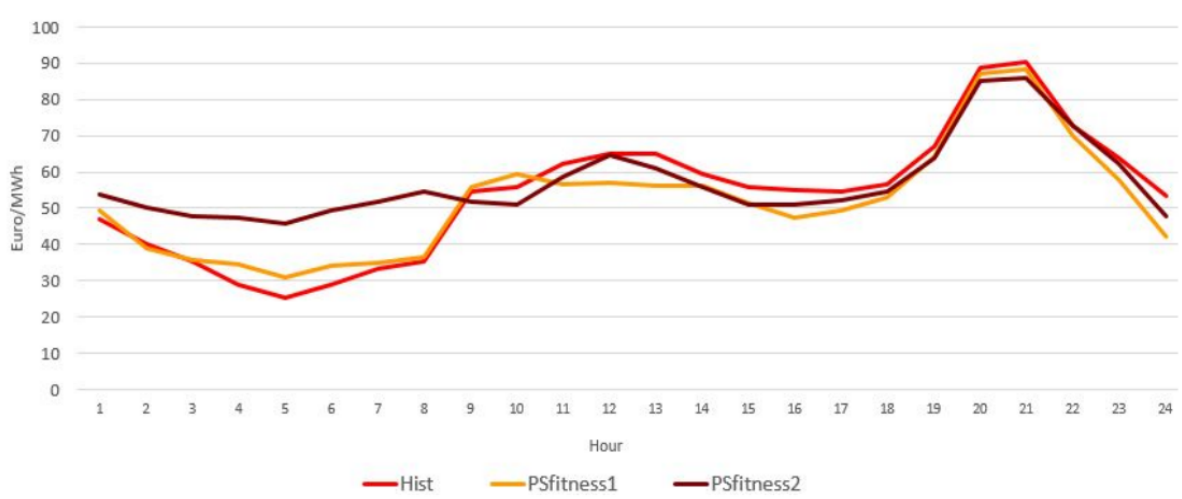

Fig. 5. Focus on the best fitness-based algorithms seen in Figure 3.

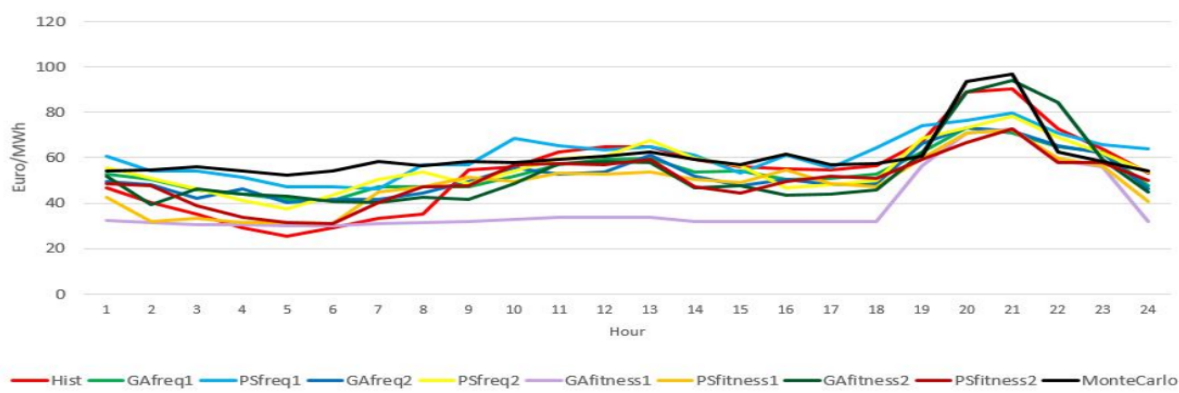

Fig. 6. Simulated PUNs for 2010-10-03, provided by IntelligentGenCo in Version 2

Both PSfreq1 (light-blue line) and PSfreq2 (yellow line) underestimate the peak hours; on the contrary, Monte Carlo (black line) is good at these hours.

Evaluation of the RMSD These considerations at the macro-level are supported by the evaluation of the root-mean-square deviation (RMSD) which is a frequently used measure of the difference between values predicted by a model and the actually observed values. The RMSD represents the sample standard deviation of the differences between predicted values and observed values. The formula we have used is the following:

$$
R M S D=\sqrt{\frac{\sum_{h=1}^{24}\left(\hat{y}_{h}-y_{h}\right)^{2}}{24}}
$$

where $\hat{y}_{h}$ and $y_{h}$ are respectively the predicted value and the observed value of the PUN at hour $h$.

Table 1 shows the RMSD of all the 9 scenarios for the two versions of the coevolutionary approach and compares them to the corresponding scenarios of two simple evolutionary approaches proposed in [2]. The Approx approach makes the 


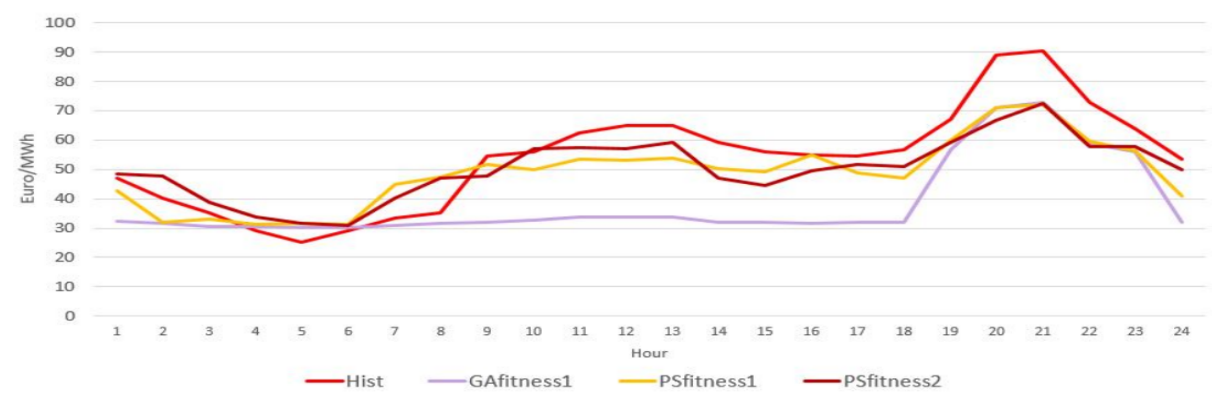

Fig. 7. Focus on the lowest algorithms seen in Figure 6.

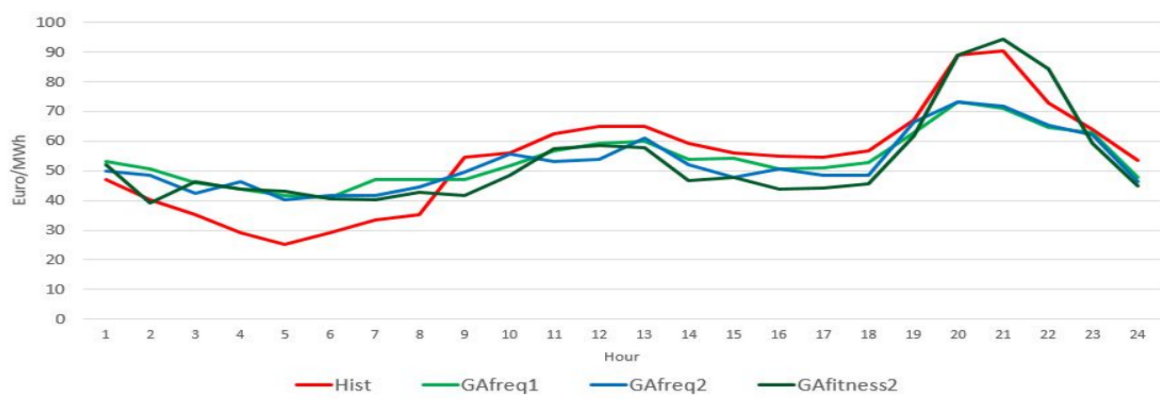

Fig. 8. Focus on the genetic algorithms seen in Figure 6.

same simplification as in [8], namely, that the vector of prices in the action space contains only one price for each collection of power plants situated in the same zone and using the same technology. The Real approach relaxes such constraint, as our co-evolutionary approach does.

We can noticed that for some optimization methods, the co-evolutionary approach gives less accurate predictions than the simple approach. This can be explained by the increased size of the optimization problem (the search space goes from just one vector of parameters to $G$ vectors of parameters, i.e. the strategy of the GenCo itself and the strategies of all its competitors). It is important to notice that the comparison to previous work has been done by allowing the same number of objective function evaluations to each approach and configuration in order for the comaparison to be fair. Nevertheless, it is interesting to

\begin{tabular}{|c|r|r|r|r|c|c|c|c|c|}
\hline Version & GAfreq1 & PSfreq1 & GAfreq2 & PSfreq2 & GAfitness1 & PSfitness1 & GAfitness2 & PSfitness2 & Montecarlo \\
\hline 1 & 9.58 & 9.87 & 21.89 & 9.83 & 18.11 & $\mathbf{4 . 9 5}$ & 22.62 & 9.97 & 19.51 \\
\hline 2 & 9.61 & 11.60 & 9.51 & 9.32 & 18.91 & 9.51 & 9.41 & 9.11 & 13.04 \\
\hline$[2]$ Approx & 8.30 & 7.74 & 18.49 & 12.12 & 18.60 & 5.14 & 35.87 & 9.29 & 14.93 \\
\hline [2] Real & 7.41 & 8.41 & 17.84 & 12.08 & 18.12 & 5.85 & 34.94 & 9.42 & 15.55 \\
\hline
\end{tabular}

Table 1. RMSD of IntelligentGenCo methods for Versions 1 and 2. 


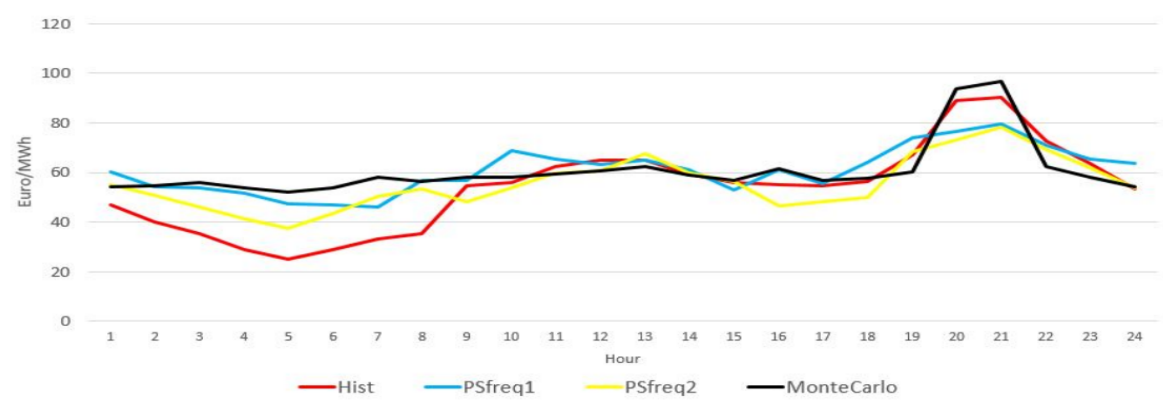

Fig. 9. Focus on selected algorithms seen in Figure 6.

notice that some optimization methods achieve improved results despite this increase in problem size. Indeed, PSfitness1 achieve the best accuracy among all tested combinations, improving over the simpler approaches of previous work. This confirms the potential of the co-evolutionary approach but also highlights the need to increase the number of objective function evaluations, if accurate predictions are sought for.

\section{Conclusion}

We have extended an existing agent-based model of the Italian electricity market and we have investigated the rationality of the market participants by comparing three optimization methods.

We can conclude that the planning for managing GenCos follows a rational strategy. It can be modeled as an optimization method using a co-evolutionary approach which better reflects the real behavior of the decision makers. We can also conclude that the particle swarm optimization method with a fitness-based strategy selection is the method which is capable to best simulate the behavior of the agents in the Italian electricity market - its results better fit the historical PUN values than all the others.

In addition to confirming the rationality of the market, our model could be used to predict the behavior of the Italian electricity market, for example by performing contingency analyses.

\section{References}

1. B. Betrò, M. Cugiani, and F. Schoen. Monte Carlo Methods in Numerical Integration and Optimization. Applied Mathematics Monographs, CNR. Giardini, Pisa, 1990.

2. S. Bevilacqua, C. da Costa Pereira, E. Guerci, F. Precioso, and C. Sartori. Analysing the impact of rationality on the italian electricity market. In Modeling Decisions for Artificial Intelligence - 16th International Conference, MDAI 2019, Milan, Italy, September 4-6, 2019, Proceedings, pages 236-247, 2019. 
3. E. Ela, C. Wang, S. Moorty, K. Ragsdale, J. OSullivan, M. Rothleder, and B. Hobbs. Electricity markets and renewables. IEEE Power and Energy Magazine, 15(27):1540-7977, 2015.

4. R. Faia, T. Pinto, and Z. A. Vale. GA optimization technique for portfolio optimization of electricity market participation. In 2016 IEEE Symposium Series on Computational Intelligence, SSCI 2016, Athens, Greece, December 6-9, 2016, pages $1-7,2016$.

5. G. Giulioni, C. Hernández, M. Posada, and A. López-Paredes. Artificial Economics: The Generative Method in Economics. Lecture Notes in Economics and Mathematical Systems 631. Springer-Verlag Berlin Heidelberg, 1 edition, 2009.

6. GME - Gestore Mercati Energetici. Data access through http://www . mercatoelettrico.org/it/download/DatiStorici.aspx.

7. D. E. Goldberg. Genetic Algorithms in Search Optimization and Machine Learning. Addison-Wesley, 1989.

8. E. Guerci, M. A. Rastegar, and S. Cincotti. Agent-based modeling and simulation of competitive wholesale electricity markets. In S. Rebennack, P. M. Pardalos, M. V. F. Pereira, and N. A. Iliadis, editors, Handbook of Power Systems II, pages 241-286. Springer-Verlag, 2010.

9. J. H. Holland. Adaptation in Natural and Artificial Systems. The University of Michigan Press, Ann Arbor, 1975.

10. J. Kennedy. Particle swarm optimization. In Encyclopedia of Machine Learning and Data Mining, pages 967-972. Springer, 2017.

11. J. Kennedy and R. Eberhart. Particle swarm optimization. In Proceedings of IEEE International Conference on Neural Networks, Part IV, pages 1942-1948, 1995.

12. G. Santos, T. Pinto, I. Praa, and Z. Vale. A new approach for multi-agent coalition formation and management in the scope of electricity markets. Energy, 36(8):5004 - 5015, 2011.

13. G. Santos, T. Pinto, I. Praa, and Z. Vale. Mascem: Optimizing the performance of a multi-agent system. Energy, 111(Supplement C):513 - 524, 2016.

14. F. Silva, B. Teixeira, T. Pinto, G. Santos, I. Praça, and Z. A. Vale. Demonstration of realistic multi-agent scenario generator for electricity markets simulation. In Advances in Practical Applications of Agents, Multi-Agent Systems, and Sustainability: The PAAMS Collection - 13th International Conference, PAAMS 2015, Salamanca, Spain, June 3-4, 2015, Proceedings, pages 316-319, 2015.

15. F. P. Sioshansi. Evolution of Global Electricity Markets. Academic Press, Boston, 2013.

16. C. Tribbia. Solving the italian electricity power exchange, 2015.

17. D. Urieli. Autonomous trading in modern electricity markets. AI Matters, 2(4):1819, 2016.

18. D. Urieli and P. Stone. Autonomous electricity trading using time-of-use tariffs in a competitive market. In Proceedings of the Thirtieth AAAI Conference on Artificial Intelligence, February 12-17, 2016, Phoenix, Arizona, USA., pages 345-352, 2016.

19. P. Vytelingum, S. D. Ramchurn, T. Voice, A. Rogers, and N. R. Jennings. Trading agents for the smart electricity grid. In 9th International Conference on Autonomous Agents and Multiagent Systems (AAMAS 2010), Toronto, Canada, May 10-14, 2010, Volume 1-3, pages 897-904, 2010. 